\title{
Prevalence of Abnormal Urodynamic Study Results in Patients with Congenital and Idiopathic Scoliosis and Its Predictive Value for the Diagnosis of Tethered Cord Syndrome: A Single Institution Clinical Study
}

\author{
Kourosh Karimi Yarandi ${ }^{1}$, Esmaeil Mohammadi ${ }^{2,3}$, Maysam Alimohammadi ${ }^{1}$, \\ Ahmad Pourrashidi Boshrabadi ${ }^{1}$, Mohammadreza Golbakhsh ${ }^{4}$, Abbas Amirjamshidi ${ }^{1}$ \\ ${ }^{1}$ Department of Neurosurgery, Sina Hospital, Tehran University of Medical Sciences, Tehran, Iran \\ ${ }^{2}$ Tehran University of Medical Sciences, Tehran, Iran \\ ${ }^{3}$ Sina Trauma and Surgery Research Center, Tehran University of Medical Sciences, Tehran, Iran \\ ${ }^{4}$ Department of Orthopedics Surgery, Sina Hospital, Tehran University of Medical Sciences, Tehran, Iran
}

\begin{abstract}
Study Design: A total of 110 patients with scoliosis were enrolled in this analytical cross-sectional study.
Purpose: We aimed to compare the urodynamic study (UDS) results of patients with idiopathic scoliosis (ISC) and congenital scoliosis (CSC) and to evaluate the clinical significance of abnormal UDS findings in predicting underlying tethered cord syndrome (TCS).

Overview of Literature: An abnormal UDS finding is commonly found in patients with CSC. However, there is no consensus regarding its prevalence in patients with ISC.

Methods: Using the STROBE checklist for cross-sectional studies, 110 patients with scoliosis were selected based on our inclusion and exclusion criteria. Among the patients, 76 presented with ISC and 34 with CSC. Demographic data and other details, such as the results of spine radiography, UDS, and magnetic resonance imaging of the spine in both supine and prone positions, were recorded and analyzed.

Results: Approximately 50\% of patients with CSC had normal UDS findings; $8.8 \%$, mild impairment; and $41.2 \%$, significant abnormalities. Moreover, $67.1 \%$ of patients with ISC had normal UDS findings; $9.2 \%$, mild impairment; and $23.7 \%$, significant abnormalities $(p=0.166)$. TCS was identified in $38.2 \%$ and $26.3 \%$ of patients with CSC and ISC, respectively $(p=0.571)$. In patients with ISC, a significantly abnormal UDS finding indicated that the risk of TCS increased from $26.3 \%$ to $50 \%$ (odds ratio [OR], 4.2; $p=0.009$ ). Meanwhile, in patients with CSC, the risk was almost similar (OR, 0.8; $p=0.8)$.

Conclusions: Even with the absence of subjective urinary symptoms, subclinical urologic impairments can be observed in a significant number of patients with ISC. An abnormal UDS finding can be a sign of underlying spinal cord tethering in a patient with ISC who is a candidate for corrective spine surgery even though it is an independent variant and is not exclusive to candidates for surgery. This finding has high clinical utility for neuro- and ortho-spine surgeons who aim to correct scoliosis (OR, 4.2; $p=0.009)$.
\end{abstract}

Keywords: Scoliosis; Tethered cord syndrome; Urodynamics; Magnetic resonance imaging

Received Sep 6, 2019; Accepted Oct 2, 2019

Corresponding author: Abbas Amirjamshidi

Department of Neurosurgery, Sina Hospital, Tehran University of Medical Sciences, Emam Ave. 1136746911 Tehran, Iran

Tel: +98-9121329864, Fax: +98-217529801, E-mail: abamirjamshidi@yahoo.com 


\section{Introduction}

Scoliosis is characterized by coronal curves of at least $10^{\circ}$ on anteroposterior (AP) spine radiograph. It is the most common form of spine deformities, excluding physiologic/degenerative kyphoscoliotic changes, and affects 3\% of the population with a female-to-male ratio of 1.4:1 [1,2]. Congenital scoliosis (CSC) and idiopathic scoliosis (ISC) are two of the most common types of scoliosis in the clinical practice of spine surgeons and are mainly observed in children, young adults, and even adults [3-5].

Spinal cord tethering is more commonly observed in patients with scoliosis than in those without [6]. The actual prevalence of tethered cord syndrome (TCS) in the different subgroups of patients with scoliosis is not fully elucidated, and the condition occurs in 5\% [7], 13\% [8], and up to $14.9 \%$ [9] of patients based on the type of scoliosis.

Urodynamic study (UDS) is an objective test that can be a sensitive tool for the evaluation of urinary function. It is a minimally invasive examination that is quite uncomfortable particularly for young children. Thus, the patients and their families must be provided with necessary information.

TCS may cause neurologic bladder and other clinical or subclinical urinary disturbances $[10,11]$. Since bladder or sphincter dysfunctions or malformations are also commonly observed in individuals with scoliosis, an abnormal UDS finding is not always associated with the presence of TCS [12]. To the best of our knowledge, the actual prevalence and significance of an abnormal UDS finding in patients with CSC and ISC are not fully elucidated. The positive and negative predictive values of the test are yet to be evaluated as well.

The preliminary findings of our study must be reported due to the following reasons: (1) to assess the prevalence of abnormal USD findings in patients with CSC and ISC who were referred to our institution, (2) to compare the findings/differences between the two groups, and (3) to emphasize that an abnormal UDS finding is common or expected in uncomplicated ISC/CSC and must be evaluated before performing major corrective spine surgeries even though there is no confirmation as to whether untethering will change the outcomes of corrective spine surgeries in either group.

Finally, based on the results, we did not aim to make a conclusion about the comparative outcomes of surgical intervention for scoliosis correction with or without cord detethering in the subpopulation of patients in this study.

\section{Materials and Methods}

\section{Study design}

The study was evaluated and approved by the ethics committee of Tehran University of Medical Sciences (IRB approval no., IR. TUMS. MEDICINE. REC. 1396.3080). A total of 110 patients with scoliosis were enrolled in this analytical cross-sectional study. Patients $(n=2)$ with complex rotary component were excluded. The STROBE statement checklist was followed meticulously (appendage). All patients were referred to our institution's neurosurgery clinic, which is a referral center for spinal deformities, between 2009 and 2017. A set of digital radiography and whole neuro-axis magnetic resonance imaging (MRI) was performed on all patients. In total, 76 patients were diagnosed with ISC and 34 with CSC. All scoliotic patients with a Cobb angle of $10^{\circ}$ or more were included. The exclusion criteria for this study were as follows: patients aged less than 5 years; those with myelomeningocele or meningocele, prior history of spine surgery, any contraindication to MRI, neuromuscular or other degenerative diseases, psychologic illnesses that affect history taking and examination reliability, paralysis, and degenerative and reactive scoliosis; and those who are unable to perform UDS due to any reasonable cause.

All participants were informed about the different steps of the study and the advantages and disadvantages of the specific evaluations, and written informed consents were obtained from the patients or their parents. Demographic data were obtained using designed datasheets, and patients were assured about data confidentiality. Any signs or symptoms of urinary malfunctions during physical examination and in the process of obtaining a patient's history were recorded.

\section{Urodynamic study}

Standard UDS was performed on each patient. Abnormal UDS finding was defined as the presence of impaired capacity/compliance (mild impairment) and overactivity of the bladder or dyssynergia (significant disorders). 
Table 1. Number of cases and distribution of age, sex, Cobb angle, and subjective sphincteric symptoms in two different types of scoliosis

\begin{tabular}{|c|c|c|c|c|}
\hline \multirow{2}{*}{ Characteristic } & \multicolumn{2}{|c|}{ Scoliosis type } & \multirow{2}{*}{ Total } & \multirow{2}{*}{$p$-value } \\
\hline & Idiopathic scoliosis & Congenital scoliosis & & \\
\hline No. of cases & $76(69.1)$ & $34(30.9)$ & $110(100.0)$ & \\
\hline Age (yr) & $17.94 \pm 7.04(8-40)$ & $11.55 \pm 5.7(3-28)$ & $15.9 \pm 7.2(3-40)$ & $<0.001$ \\
\hline Sex & & & & 0.053 \\
\hline Male & $22(28.9)$ & $16(47.1)$ & $38(34.5)$ & \\
\hline Female & $54(71.1)$ & $18(52.9)$ & $72(65.5)$ & \\
\hline Male:female ratio & $1: 2.4$ & $1: 1.1$ & $1: 1.8$ & \\
\hline Subjective sphincteric symptoms & & & & 0.31 \\
\hline Yes & $6(7.9)$ & $5(14.7)$ & $11(10.0)$ & \\
\hline No & $70(92.1)$ & $29(85.3)$ & $99(90.0)$ & \\
\hline Cobb angle & $68.4 \pm 28.3(10-120)$ & $55.3 \pm 22.8(10-160)$ & $64.4 \pm 27.3(10-160)$ & 0.022 \\
\hline
\end{tabular}

Values are presented as number (\%) or mean \pm standard deviation (range).

\section{Evaluation of tethered cord syndrome}

Whole neuro-axis MRI with and without contrast enhancement was performed on all participants to assess the type of scoliosis, intracanal and cord anomalies, and position of the tip of the conus medullaris. The MRI images were obtained with the 1.5 Tesla Siemens MRI equipment. Furthermore, the presence of anomalies, such as syrinx, intradural lipoma, different types of split cord malformations (SCMs), and thick filum terminale, was cautiously evaluated and recorded. We also identified patients with cord tethering abnormality, which was defined as the presence of at least one of the following: a low-lying filum terminale at any place below the L2/L3 disk space, neurologic manifestations of cord tethering and placement of the tip of the filum at any level below L1/L2, SCM I, or any other tethering pathologies of the spine, including the dermal sinus tract or tumors, or a tight filum terminale on MRI. The presence of a tight filum terminale was further evaluated by performing MRI on all patients while in prone position. A forward movement of the canal width by at least $10 \%$ was considered normal, thereby ruling out the tightness of the filum terminale [13]. The presence of cord tethering or tightness of the filum terminale was further confirmed during surgery by two skilled neurosurgeon observers for each case. Examination of the tightness of the filum and stretch test were routinely performed during surgery; after a consensus was achieved between the observers, these patients were considered to have tethered cords. Furthermore, AP and lateral standing whole-spine radiography and bending X-ray images were obtained to evaluate for Cobb angle. Two neurosurgeons performed all physical examinations, and the imaging data were analyzed by two radiologists who are experienced in evaluating MRI and spine radiography findings. The UDS findings were reviewed by an expert urologist. All examiners were blinded to the other data of the cases during observation.

\section{Statistical analysis}

All statistical analyses were performed using the IBM SPSS software ver. 22.0 (IBM Corp., Armonk, NY, USA). Categorical variables were presented as absolute (numbers) and relative (percent) values and continuous variables as mean and standard deviation. The UDS results, known as a categorical variable, were tested for an association with demographic characteristics, type of scoliosis, sphincter problems, spine MRI anomalies, and other variables using chi-square analysis for categorical variables. Independent sample $t$-test was used for comparing continuous variables. A $p$-value $<0.05$ was considered statistically significant.

\section{Results}

\section{Characteristics of the participants}

A total of 110 patients with scoliosis who met our criteria were assessed. Among the participants, 72 (65.5\%) 
Table 2. UDS results according to the type of scoliosis

\begin{tabular}{|c|c|c|c|c|}
\hline \multirow{2}{*}{ Variable } & \multicolumn{3}{|c|}{ Scoliosis type } & \multirow{2}{*}{$p$-value } \\
\hline & Idiopathic scoliosis & Congenital scoliosis & Total & \\
\hline Normal & $51(67.1)$ & $17(50.0)$ & $68(61.8)$ & 0.166 \\
\hline Mild UDS impairment: decreased compliance or capacity only & $7(9.2)$ & $3(8.8)$ & $10(9.1)$ & \\
\hline $\begin{array}{l}\text { Significant UDS impairment: overactive bladder or hyperactive } \\
\text { detrusor and/or detrusor dyssynergia }\end{array}$ & $18(23.7)$ & $14(41.2)$ & $32(29.1)$ & \\
\hline Total & $76(100.0)$ & $34(100.0)$ & $110(100.0)$ & \\
\hline
\end{tabular}

Values are presented as number (\%).

UDS, urodynamic study.

Table 3. UDS results in patients without sphincteric symptoms, according to the type of scoliosis

\begin{tabular}{lccc} 
Variable & \multicolumn{3}{c}{ Scoliosis type } \\
\cline { 2 - 3 } & Idiopathic scoliosis & Congenital scoliosis & Total \\
Normal UDS & $48(68.6)$ & $16(55.2)$ & $64(64.6)$ \\
Mild UDS impairment: decreased compliance/ capacity & $7(10.0)$ & $1(3.4)$ & $8(8.1)$ \\
$\begin{array}{l}\text { Significant UDS impairment: overactive bladder or hyperactive detrusor } \\
\quad \text { and/or detrusor dyssynergia }\end{array}$ & $15(21.4)$ & $12(41.4)$ & $27(27.3)$ \\
\hline Total & $70(100.0)$ & $29(100.0)$ & $99(100.0)$ \\
\hline
\end{tabular}

Values are presented as number (\%).

UDS, urodynamic study.

were women and $38(34.5 \%)$ men with a ratio of $1.8: 1$ $(p=0.053)$ (Table 1). Moreover, 34 patients (30.9\%) presented with CSC and 76 (69.1\%) with ISC. The mean ages were $11.55 \pm 5.7$ years (range, $3-28$ years) in the congenital group and $17.94 \pm 7.04$ years (range, $8-40$ years) in the idiopathic group $(p<0.001)$.

\section{Urodynamic study results}

UDS was performed on all participants. A total of 68 patients (61.8\%) had normal UDS findings. Significant problems, such as dyssynergia and overactive bladder, were noted in 32 patients (29.1\%), and milder problems, including low bladder capacity and compliance, were observed in 10 patients (9.1\%) (Table 2). Moreover, 11 (10\%) of 110 patients had subjective urinary sphincter problems. The presence of such symptoms was more common in patients with CSC than in those with ISC. However, the result was not significantly different $(p=0.31$ ) (Table 1). Among 99 patients (90\%) without sphincteric symptoms, 64 (64.6\%) had normal UDS finding; 8 (8.1\%), mild problems (only changes in capacity/compliance); and 27 (27.3\%), significant UDS disorders. Among the 27 patients with significant UDS abnormalities, 12 presented with CSC and 15 with ISC. No significant correlation was observed between the type of scoliosis and changes in UDS findings ( $p=0.098$ ) (Table 3).

\section{Imaging results}

Standard AP digital radiograph images of the whole spine were used to determine the Cobb angle. The mean angle in the entire sample was $64.4^{\circ} \pm 27.3^{\circ}$, with $10^{\circ}$ being the lowest and $160^{\circ}$ the highest. The mean values for the Cobb angles were $55.3^{\circ} \pm 22.8^{\circ}$ in the congenital group and $68.4^{\circ} \pm 28.3^{\circ}$ in the idiopathic group $(p=0.022)$ (Table 1$)$.

TCS or anomalies that could cause cord tethering were found on the MRI images of $26.3 \%$ and $38.2 \%$ of patients with ISC and CSC, respectively. Although TCS was more commonly found in the congenital group than in the idiopathic group, the result was not significantly different $(p=0.571)$. This finding is important in this research (Table $4)$.

\section{Combination of urodynamic study and magnetic resonance imaging findings}

In the ISC group, a significantly abnormal UDS finding 
Table 4. Spine magnetic resonance imaging and physical exam findings in different types of scoliosis

\begin{tabular}{|c|c|c|c|}
\hline Variable & Idiopathic scoliosis & Congenital scoliosis & Any type of scoliosis \\
\hline \multicolumn{4}{|l|}{ TCS or cord tethering ${ }^{\mathrm{a})}$} \\
\hline Low-lying conus medullaris ${ }^{\text {b) }}$ & $15(19.7)$ & $8(23.5)$ & $23(20.9)$ \\
\hline SCM type I (diasthematomyelia) ${ }^{c}$ & $1(1.3)$ & $1(2.9)$ & $2(1.8)$ \\
\hline Sinus tract ${ }^{\mathrm{c})}$ & $1(1.3)$ & $1(2.9)$ & $2(1.8)$ \\
\hline Tight filum terminale & $2(2.6)$ & $1(2.9)$ & $3(2.7)$ \\
\hline Thick fatty filum terminale & $1(1.3)$ & $2(5.8)$ & $3(2.7)$ \\
\hline Tumors & 0 & 0 & 0 \\
\hline Sub-total & $20(26.3)$ & $13(38.2)$ & $33(30.0)$ \\
\hline Completely normal & $52(68.4)$ & $19(55.8)$ & $71(64.5)$ \\
\hline Only syrinx & $4(5.2)$ & $1(2.9)$ & $5(4.5)$ \\
\hline SCM type II (diplomyelia) ${ }^{d /}$ & 0 & $1(2.9)$ & $1^{\text {d) }}(0.9)$ \\
\hline Syrinx+SCM type II & 0 & 0 & 0 \\
\hline Sub-total & $56(73.6)$ & $21(61.7)$ & $77(0.7)$ \\
\hline Total & $76(100.0)$ & $34(100.0)$ & $110(100.0)$ \\
\hline$p$-value & \multicolumn{2}{|c|}{0.571} & \\
\hline
\end{tabular}

Values are presented as number (\%).

TCS, tethered cord syndrome; SCM, split cord malformation.

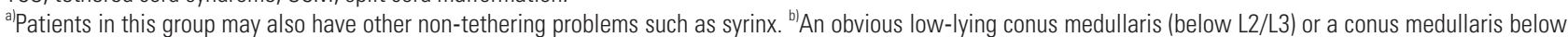
L1/L2 with significant signs of cord tethering in physical examination. ${ }^{\text {cl} C o n c o m i t a n c e ~ o f ~ t h i s ~ p r o b l e m ~ a n d ~ a ~ l o w-l y i n g ~ c o n u s ~ m e d u l l a r i s ~ i s ~ a l s o ~ i n c l u d e d . ~}{ }^{\text {d) }}$ Altogether, four cases of SCM type II were recognized but three were included in TCS group because of co-existence with another anomaly that caused tethering.

Table 5. Predictive value of UDS for diagnosis of tethered cord

\begin{tabular}{|c|c|c|c|c|c|c|c|c|c|}
\hline \multirow{2}{*}{ Variable } & \multicolumn{3}{|c|}{ Any type of scoliosis } & \multicolumn{3}{|c|}{ Idiopathic scoliosis } & \multicolumn{3}{|c|}{ Congenital scoliosis } \\
\hline & Non-TCS & TCS & Total & Non-TCS & TCS & Total & Non-TCS & TCS & Total \\
\hline \multicolumn{10}{|l|}{ UDS } \\
\hline Normal or mild impairment & $59(75.6)$ & $19(24.4)$ & $78(100.0)$ & $47(81.0)$ & $11(19.0)$ & $58(100.0)$ & $12(60.0)$ & $8(40.0)$ & $20(100.0)$ \\
\hline Significant impairment & $18(56.2)$ & $14(43.8)$ & $32(100.0)$ & $9(50.0)$ & $9(50.0)$ & $18(100.0)$ & $9(64.3)$ & $5(35.7)$ & $14(100.0)$ \\
\hline Total & $77(70.0)$ & $33(30.0)$ & $110(100.0)$ & $56(73.7)$ & $20(26.3)$ & $76(100.0)$ & $21(61.8)$ & $13(38.2)$ & $34(100.0)$ \\
\hline Odds ratio (95\% confidence interval) & \multicolumn{3}{|c|}{$2.41(1.01-5.75)$} & \multicolumn{3}{|c|}{$4.2(1.37-13.27)$} & \multicolumn{3}{|c|}{$0.8(0.2-3.42)$} \\
\hline$p$-value & \multicolumn{3}{|c|}{0.04} & \multicolumn{3}{|c|}{0.009} & \multicolumn{3}{|c|}{0.8} \\
\hline
\end{tabular}

Values are presented as number (\%).

UDS, urodynamic study; TCS, tethered cord syndrome.

indicated that the risk of TCS increased from $26.3 \%$ to $50 \%$ (odds ratio [OR], 4.2). Meanwhile, in the congenital group, the risk was similar: $35.7 \%$ in patients with a significantly abnormal UDS finding and $38.2 \%$ in patients with CSC (OR, 0.8) (Table 5). The predictive power of a significantly abnormal UDS finding for the presence of TCS was statistically significant $(p=0.009$ ) in the idiopathic group. This value was high enough to be considered significant in all patients with scoliosis $(p=0.04)$, although it was completely irrelevant in predicting TCS in the congenital group $(p=0.8)$.

During follow-ups, the patients were re-evaluated by a multidisciplinary panel, which included neurosurgeons, ortho-spine surgeons, neuroradiologists, and urologists, to plan for further corrective spine surgery. 


\section{Discussion}

Developmental problems play a significant role in the etiology of CSC. Other congenital problems, such as cord anomalies and urinary tract abnormalities, are also commonly observed in this group of patients [3]. Therefore, these patients must be further assessed for underlying problems, with consideration of subclinical or important concomitant abnormalities [6]. We hypothesized that urinary problems and spinal cord anomalies, such as TCS, are frequently observed in patients with ISC. Thus, the present study aimed to describe the prevalence of such problems in patients with ISC, compare the prevalence of these problems between patients with ISC and CSC, and evaluate the association between an abnormal UDS finding and cord tethering in patients with ISC and SCS to prevent possible cord damage due to surgical interventions.

Almost $70 \%$ of our patients had ISC, which is comparable to the report of Winter [5] in 1995 showing that $80 \%$ of their participants had the condition. The mean age of patients with CSC was significantly lower than that of patients with ISC $11.55 \pm 5.7$ years versus $17.94 \pm 7$ years $(p<0.001)$. This finding is consistent with that of previous studies reporting that patients with CSC were younger than those with ISC [3]. Our patients had a wider age range from childhood up to the late 20 s and even later in adulthood. Meanwhile, in other studies, most patients present with such condition between infancy and adolescence. This phenomenon can be attributed to low socioeconomic status, which is still observed in some regions despite recent developments. In addition, as previously noted, patients younger than 5 years were excluded from our study because the UDS results might be unreliable in such cases. The female-to-male ratios were 2.4:1 in patients with ISC and 1.1:1 in patients with CSC, and the result was significantly different between the two groups $(p=0.053)$. These findings are consistent with those of previous reports about ISC showing a female-to-male ratio of 2:1 in younger patients and 3:1 in older ones [14]. Our results reflect the wider age range distribution in the present study. In addition, the female-to-male ratio in patients with CSC has been as low as 1.2:1, which is similar to our finding [9]. The mean Cobb angles were $55.3^{\circ} \pm 22.8^{\circ}$ in the congenital group and $68.4^{\circ} \pm 28.3^{\circ}$ in the idiopathic group. The difference was statistically significant $(p=0.022)$. In another study in the literature, a higher Cobb angle was observed in patients with ISC than in those with CSC [15].

The UDS results were categorized as normal, mild (lower than normal compliance or capacity), and significant (detrusor overactivity or dyssynergia) impairments. Most patients with ISC (67.1\%) had normal UDS findings. On the other hand, in CSC, at least one significant type of impairment on UDS was found in $41.2 \%$ of the cases. Notably, although normal UDS results are more commonly observed in patients with ISC than in those with CSC, the difference was statistically insignificant $(p=0.166)$.

Traditionally, UDS is only performed on patients with urinary symptoms, mainly to document existing urinary abnormalities [6]. Our study confirmed that UDS can identify possible important subclinical urinary problems in scoliotic patients without clinically significant urinary problems but of value in the primary evaluation of scoliotic patients. To assess the rate of subclinical urinary problems better in each type of scoliosis, we analyzed the UDS results of patients without urination problems separately. In our study, $44.8 \%$ of patients in the congenital group and $31.4 \%$ in the idiopathic group had at least one type of urinary problem, which was detectable in UDS. However, their sphincter function was clinically normal. This finding indicated that the absence of clinical symptoms is not a reliable indicator in ruling out urinary problems in scoliosis and UDS can help with these problems. A statistically significant difference was observed between the two groups. However, our study confirmed that the rate of subclinical urinary problems is high enough in idiopathic cases. Thus, a study with larger sample size might help identify any significant difference $(p=0.098)$ (Table 3).

The evaluation of spinal cord MRI, physical examination, and intraoperative findings revealed that $38.2 \%$ of patients with CSC and $26.3 \%$ with ISC had at least one type of cord tethering pathology. However, the difference was statistically insignificant $(p=0.571)$. The presence of an abnormal UDS finding in our series was a predictor of cord tethering in idiopathic cases, which indicated that the risk of TCS increased from $26.3 \%$ to $50.0 \%$. However, it was not a risk factor for the presence of TCS in the congenital group in our series (Table 5). This result indicated that performing UDS may help in the diagnosis of TCS in specific patients with ISC. However, it may not be useful in patients with CSC. An abnormal UDS finding can result in the clinical suspicion of an underlying cord tethering pathology, which is remarkable in the evaluation of a patient with ISC. Since an abnormal UDS may be 
attributed to a congenital developmental anomaly in the genitourinary system, the mere presence of an abnormal UDS finding in a patient with CSC might not be related to an underlying problem in the spinal cord.

In summary, a significantly abnormal UDS finding has a good predictive value for TCS in ISC (OR, 4.2; 95\% confidence interval [CI], 1.37-13.27; $p=0.009)$. However, it might be useless in predicting TCS in CSC (OR, 0.8; 95\% CI, $0.2-3.42 ; p=0.8)$. The difference was remarkable in the idiopathic group, thereby resulting in a significant difference in all cases of scoliosis (OR, 2.41; 95\% CI, 1.01-5.75; $p=0.04$ ). This difference should not be misinterpreted as the value of UDS in predicting TCS in all cases of scoliosis because our data indicated that this test is almost not beneficial in CSC.

A difference was observed between the incidences of TCS in our series and previous studies. Erfani et al. [8] in 2008 showed that the condition occurs in $13 \%$ of patients with CSC. Meanwhile, SCM type I was observed in 17.4\% of patients [8]. The authors did not specify whether an overlap was observed between the two groups, considering that SCM type I can also cause cord tethering, and the incidence rate of such overlap was not determined in that report. Ghandhari et al. [9] in 2015 showed that TCS has an incidence rate of 3\%. Moreover, $14.9 \%$ of patients have SCM type I. These two conditions were analyzed separately in our study, and results showed that the rate of lowlying conus medullaris was significantly higher than that of SCM type I. Based on our experience, low-lying conus medullaris was more commonly observed than SCM type I in patients with scoliosis. Pahys et al. [7] in 2009 reported that $5 \%$ of patients with infantile ISC present with concomitant TCS. In the current study, the incidence rate of TCS was significantly higher in patients with ISC, which might be attributed to the varying age distributions of the patients. Moreover, we included all causes of spinal cord tethering, such as low-lying conus medullaris, SCM type I (diastematomyelia), sinus tract, tight filum terminale, and thick fatty filum terminale. In addition, the patients underwent MRI while in prone position, which is considered an additional examination for the diagnosis of tight filum terminale. These factors may explain the higher rate of spinal cord tethering found in our study.

No infantile cases were enrolled in this study, and as a result of referring cases to the hospital, selection bias might exist. Moreover, this study was conducted in a single institution, and the sample size was relatively small.
Thus, further large multi-institutional studies must be conducted to validate the results of the present study, and the inclusion of an age-matched control group without scoliosis can increase the power of this study. Finally, a cost-effectiveness analysis can be included in studies with a larger sample size.

\section{Conclusions}

Even in the absence of subjective urinary symptoms, subclinical urologic impairments can be observed in a significantly high number of scoliotic patients. The difference is marginally significant between patients with ISC and CSC.

The correlation between abnormal UDS findings and the abnormalities causing spinal cord tethering is more significant in patients with ISC. Thus, UDS may be performed as additional examination for the evaluation of TCS in selected patients within this subgroup. However, individuals who can benefit from such approach are yet to be identified via larger-scale studies that evaluate costs and benefits. In contrast, an abnormal UDS finding in patients with ISC may have no predictive value for the presence of TCS. Thus, further studies must aim to evaluate the positive and negative predictive values of UDS for the outcome of surgery.

\section{Conflict of Interest}

No potential conflict of interest relevant to this article was reported.

\section{Acknowledgments}

The authors would like to thank Epidemiology and Biostatistics Department of the Research Development Center of Sina Hospital for their technical assistance. The authors would like to thank Glareh Amirjamshidi. Ph.D., P.Eng., for carefully revising the manuscript for the English Language.

\section{References}

1. Mohammadi P, Akbari M, Sarrafzadeh J, Moradi Z. Comparison of respiratory muscles activity and exercise capacity in patients with idiopathic scoliosis and healthy individuals. Physiother Theory Pract 
2014;30:552-6.

2. Yaman O, Dalbayrak S. Idiopathic scoliosis. Turk Neurosurg 2014;24:646-57.

3. Burnei G, Gavriliu S, Vlad C, et al. Congenital scoliosis: an up-to-date. J Med Life 2015;8:388-97.

4. Shakil H, Iqbal ZA, Al-Ghadir AH. Scoliosis: review of types of curves, etiological theories and conservative treatment. J Back Musculoskelet Rehabil 2014;27:111-5.

5. Winter RB. The surgical treatment of congenital spine deformity: general principles and helpful hints. Iowa Orthop J 1995;15:79-94.

6. Lew SM, Kothbauer KF. Tethered cord syndrome: an updated review. Pediatr Neurosurg 2007;43:236-48.

7. Pahys JM, Samdani AF, Betz RR. Intraspinal anomalies in infantile idiopathic scoliosis: prevalence and role of magnetic resonance imaging. Spine (Phila $\mathrm{Pa}$ 1976) 2009;34:E434-8.

8. Erfani MA, Ganjavian MS, Ameri E, Namazi H, Solooki S. Occult intraspinal abnormalities and congenital scoliosis. J Res Med Sci 2007;12:53-7.

9. Ghandhari H, Tari HV, Ameri E, Safari MB, Fouladi DF. Vertebral, rib, and intraspinal anomalies in congenital scoliosis: a study on 202 Caucasians. Eur Spine J 2015;24:1510-21.
10. Alzahrani A, Alsowayan O, Farmer JP, Capolicchio JP, Jednak R, El-Sherbiny M. Comprehensive analysis of the clinical and urodynamic outcomes of secondary tethered spinal cord before and after spinal cord untethering. J Pediatr Urol 2016;12:101.

11. Alsowayan O, Alzahrani A, Farmer JP, Capolicchio JP, Jednak R, El-Sherbiny M. Comprehensive analysis of the clinical and urodynamic outcomes of primary tethered spinal cord before and after spinal cord untethering. J Pediatr Urol 2016;12:285.

12. Nogueira M, Greenfield SP, Wan J, Santana A, Li V. Tethered cord in children: a clinical classification with urodynamic correlation. J Urol 2004;172(4 Pt 2):1677-80.

13. Stamates MM, Frim DM, Yang CW, Katzman GL, Ali $\mathrm{S}$. Magnetic resonance imaging in the prone position and the diagnosis of tethered spinal cord. J Neurosurg Pediatr 2018;21:4-10.

14. Konieczny MR, Senyurt H, Krauspe R. Epidemiology of adolescent idiopathic scoliosis. J Child Orthop 2013;7:3-9.

15. Owange-Iraka JW, Harrison A, Warner JO. Lung function in congenital and idiopathic scoliosis. Eur J Pediatr 1984;142:198-200. 